\title{
Affinity Profiles Categorize RNA-Binding Proteins into Distinctive Groups
}

\author{
Naomi Ueda, Riki Kurokawa* \\ Division of Gene Structure and Function, Research Center for Genomic Medicine, Saitama Medical University, Saitama, Japan
}

Email address:

rkurokaw@saitama-med.ac.jp (R. Kurokawa)

${ }^{*}$ Corresponding author

\section{To cite this article:}

Naomi Ueda, Riki Kurokawa. Affinity Profiles Categorize RNA-Binding Proteins into Distinctive Groups. Biomedical Sciences. Vol. 4, No. 3, 2018, pp. 24-31. doi: 10.11648/j.bs.20180403.11

Received: October 15, 2018; Accepted: October 31, 2018; Published: November 27, 2018

\begin{abstract}
Recently, we have established a novel assay to detect interaction of RNA-binding proteins (RBPs) with RNA, using biotinylated RNA oligos to capture RBPs with Western blot of specific antibodies against RBPs. The assay detects RNA binding more confidently than the traditional gel shift assay. Starting with completely randomized RNA oligos from 5mer through 12mer length, their binding was examined with HeLa cell nuclear extract (NE). Coomassie brilliant blue-based (CBB) staining did not detect any strong signal. Western blot analysis of typical six RBP antibodies showed four RBPs bound with the random RNA oligos. hnRNPUL2 bound to all from 5mer to 12mer of RNA oligos, while no TLS and hnRNPH1 signal was detected in the random RNA oligo samples. Next, base specificity was examined using sets of oligos of RNA fixed at G, A, U, and C (GAUC RNA oliogs). The RNA oligos fixed at "G" (G RNA oligos) have the most prominent protein bands. A, U, and C of RNA oligos were shown to bind less numbers of protein. Western blot indicated that hnRNPUL2 and hnRNPU bind all four oligos of GAUC at the 10mer length. Contrarily, TLS and hnRNPH1 have no binding with these oligos of GAUC. Then, poly $\mathrm{G}, \mathrm{A}, \mathrm{U}$ and $\mathrm{C}$ of RNA at the length of $100 \mathrm{mer}$ were tested to see binding profile of RBPs. The CBB staining of the fractions bound with these four polymers of RNA showed that more bands were bound than GAUC RNA oligos. hnRNPU bound well to poly G, A, and, U, but slightly less to poly C. Intriguingly, TLS and hnRNPH1 have binding only to poly G, and also to their common specific sites consisting of GGUG motifs. These data demonstrate that RNA binding is regulated with three factors, length, base composition, and sequence. Furthermore, hnRNPU and hnRNPUL2 have low specificity binding to RNAs, while TLS and hnRNPH1 exert high specific binding. These different propensities in bindings of RBPs are supposed to support specific biological roles in living cells.
\end{abstract}

Keywords: TLS, FUS, Random RNA Oligos, hnRNPH1, hnRNPU, hnRNPUL2, DDX21, hnRNPAB

\section{Introduction}

Physiology of RNA has been generating more impact on modern biomedical sciences extending the previous views for the RNA metabolism that has been just oriented to steps of the process of the Central Dogma [1-10]. The intensive investigations on transcriptomes of the human genome-derived RNAs have uncovered enormous numbers of RNAs transcribed from the noncoding regions [11-13]. Majority of the unidentified transcripts are found to be unannotated long noncoding RNAs (lncRNAs) of which biological activities remain largely unidentified [14], although their activities are supposed to be mediated by
RNA-binding proteins. RNA binding is much more intricate mechanism than DNA binding. RBPs are characterized by proficiency of RNA perception and possess divergent capability in living cells. Elucidation of mechanism of generating specificity of RBPs will give rise to developing more efficient technology to dissect them. Depending on technology in detection, experimental data indicate distinctive propensity.

RBPs form ribonucleoprotein (RNP) complexes primarily involving in regulation of gene expressions. RBPs exert their functions by interacting with RNAs via previously identified 
RNA binding structures of RBPs [15], such as RNA recognition motif (RRM) [16], and DEAD box helicase domain [17]. However, prompt progress in exploring the structures of large RNP complexes like ribosome [18-20] and spliceosome [21, 22] indicate unprecedented interactions between proteins and RNAs without canonical RNA binding domains. These observations suggest that unconventional RNA bindings are more general events than previously perceived.

Most of RBPs possess intrinsically disordered regions (IDRs) or low complexity domains (LCDs) in their amino acid sequences [23]. These domains have been found to play critical roles in targeting RBPs into cellular bodies such as RNA granules and paraspeckles [24-26]. Their dynamic composition and amorphous structure still remain unsolved. Some RBPs have KH-rich peptide regions located in LCDs. These motifs possibly interact with RNA [27]. These observations indicate that LCD should be a target for RNA, suggesting that previously unidentified amino acid sequence functions as RNA binding motif.

There should be possible unexpected RBPs with novel RNA binding motif consisting of LCD in living cells. Most certain criterion for RBPs is just to detect binding ability to RNA. Recently, we have established a novel assay to detect binding of RNA-binding proteins, using biotinylated RNA oligos to capture RBPs with Western blot of specific antibodies against RBPs $[28,29]$. The assay more confidently detects RNA binding than the traditional gel shift assay. For extensive analysis of the RNA bindings in RBPs, we start to categorize the RNA bindings according to their affinity to RNAs using our assay system. This sort of categorizing will give us an expedient technology to dissect RNA binding and be led to fruitful direction of the future investigation of RBPs.

\section{Materials and Methods}

\subsection{Antibodies and Reagents}

Mouse anti-TLS/FUS antibody (611385, Lot no.2209827) was purchased from BD Biosciences. Rabbit anti-hnRNPU antibody (ab20666, Lot no.825937) was purchased from Abcam. Rabbit anti-hnRNPUL2 antibody (A304-619A-T, A304-619A-T-1) was purchased from BETHYL. Rabbit anti-DDX21 antibody (ab126968, Lot no. GR166655-4) was purchased from Abcam. Rabbit anti-hnRNPAB antibody (AP5522a, Lot no. SA100907AO) was purchased from ABGENT. Rabbit anti-mouse HRP-conjugated IgG (P0161, 20017456) was purchased from Dako. Goat anti-rabbit HRP-conjugated IgG (7074S, 25) was purchased from Cell signaling. The RNase inhibitor (SIN-201) was purchased from Toyobo Life Science. HeLa cell NE was prepared as previously described protocol [29-31].

\subsection{RNA Binding Assay}

The RNA binding assays were performed previously described [28]. Briefly, it has shown below. DynabeadsM280 (Thermo Fisher) was washed with PBS containing $0.02 \%$ Tween 20 . Ten pmol of biotinylated RNA oligos or RNAs was added to the beads and incubated for $15 \mathrm{~min}$ at room temperature with rotation. Subsequently, beads were incubated with HeLa cell NE for $1 \mathrm{~h}$ at $4^{\circ} \mathrm{C}$ with rotation. Beads were then washed three times in $1 \mathrm{ml}$ of WCE buffer and resuspended in SDS sample buffer and boiled at $100^{\circ} \mathrm{C}$ for $2 \mathrm{~min}$. Finally, the Dynabeads were removed and supernatants were applied to SDS-poly acrylamide gel following for stain with the SimplyBlue ${ }^{\mathrm{TM}}$ SafeStain (Thermo Fisher) or Western blotting analyses.

\subsection{Protein Analysis}

SDS-polyacrylamide gel electrophoresis was performed with $10 \%$ gels following coomassie brilliant blue staining [29]. Western blotting was done with anti-TLS monoclonal antibody from the BD bioscience, 611385 with the dilution ratio 1:2000 using standard protocol shown previously [29].

\section{Results}

Recently, we have established simple and solid technique of detection of the binding of RBPs, using biotinylated RNA oligos to precipitate RBPs by detection with Western blot of the antibodies against RBPs. This RBP assay more firmly detects RNA binding compared to gel shift assay with ${ }^{32}$ P-labeled RNA oligos.

Using completely randomized RNA oligos from $5 \mathrm{mer}$ through $12 \mathrm{mer}$, their binding was examined with proteins of HeLa cell NE (Figure 1A). These RNA oligos are usually employed to be negative control of typical RNA binding experiment. CBB-based protein staining did not detect any strong signal although some faint bands appeared (Figure 1A). Unexpectedly, the Western blot analysis of antibodies against typical six RBPs showed four RBPs bound with the random RNA oligos. All RNA oligos from 5mer to $12 \mathrm{mer}$ RNA were bound with hnRNPUL2, while hnRNPU bound RNA oligos from 6 mer to 12 mer (Figure 1B). These two RBPs exert minimal specificity so far tested. hnRNPAB bound to RNA oligos from 7 mer to 11 mer while DDX21 bound to RNA oligos from 9mer to 12 mer (Figure 1C). hnRNPAB alone shows preferred binding to specific length of RNA oligos. Any TLS and hnRNPH1 signal was detected in these random RNA oligo samples (data not shown). 


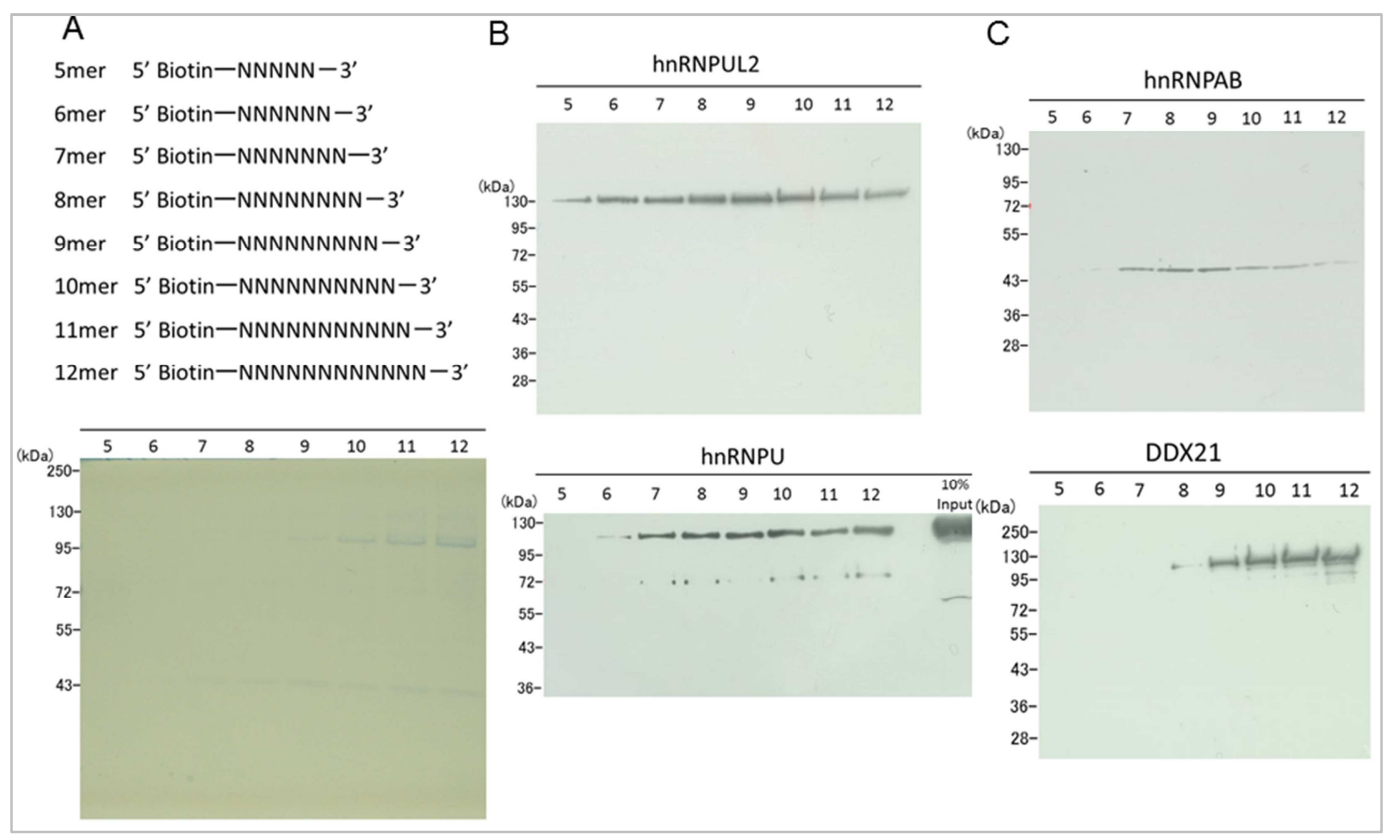

Figure 1. Low specificity binding of RNA binding proteins.

(A) Upper panel: Randomized RNA oligos are examined. Lower panel: Fifty $\mu$ ls of NE were tested for binding with the depicted randomized RNA oligos, and detected with CBB-staining. " 5 " represents "5mer", and so on.

(B) Low specificity binding to random RNA oligos.

hnRNPUL2 binds random RNA oligos that are longer than 5mers. 10\% input was at the similar intensity as hnRNPU. hnRNPU binds random RNA oligos that are larger than 7 mers.

(C) Moderate specificity binding to RNA oligos.

hnRNPAB and DDX21 bind RNA oligos with relatively narrow ranges of their length. 10\% inputs were at the similar intensity as hnRNPU in Figure 1B.

Next, base specificity of RBPs was examined using sets of oligos of RNA fixed their base compositions at $\mathrm{G}$ (Guanine), A (Adenine), U (Uracil), and C (Cytosine) (GAUC RNA oliogs). Oligomers, from 5mer, to 11 mer of G RNA oligos were bound with the most prominent protein bands (Figure 2A). The same sets of $A, U$, and $C$ of RNA oligos were bound to less numbers of proteins (Figure 2A). Based on scanning the oligos from $5 \mathrm{mer}$ to $11 \mathrm{mer}$, we set the $10 \mathrm{mer}$ as a representative length of the RNA oligo sets. Western blot showed that hnRNPUL2 and hnRNPU bind all four oligos of GAUC at the 10mer length (Figure 2B). DDX21 bound G and $U$ at 10 mer while, hnRNPAB binds only a $U$ at $10 \mathrm{mer}$, indicating base preference on these two RBPs (Figure 2C). TLS and hnRNPH1 have no binding with these oligomers of GAUC (data not shown).

For further RNA binding assay, we employed poly G, poly $\mathrm{A}$, poly $\mathrm{U}$, and poly $\mathrm{C}$ which have approximately $100 \mathrm{mer}$ length of RNA to evaluate impact of the length of RNA. CBB staining of the fractions bound with these four polymers of RNA indicated that much more bands than oligomers in Figure 2 (Figure 3A). Especially, poly $\mathrm{G}$ and poly $\mathrm{U}$ were shown to have comparable numbers of bands, while poly $\mathrm{A}$ and poly $\mathrm{C}$ were found to have relatively smaller numbers of bands (Figure 3A). Western blot revealed that hnRNPU binds well to poly $\mathrm{G}, \mathrm{A}$, and, $\mathrm{U}$, but less to poly C (Figure 3B). hnRNPUL2 and DDX21 indicated a similar binding profile to all four polymer RNAs with less intensity than hnRNPU (Figure $3 \mathrm{~B}$ ). hnRNPAB has preferred binding to poly $\mathrm{G}, \mathrm{A}$, and U. but no binding to poly C (Figure 3C). Interestingly, hnRNPH1 and TLS were exclusively bound to poly G (Figure 3D).

Previously, we have identified specific RNA binding site for TLS in a long noncoding RNA, pncRNA-D, which is transcribed from the promoter for cyclin D1 gene [30]. The TLS-target RNA sequence at 32 to 44 of pncRNA-D is designated as $5^{\prime}(1-1)$, and the adjacent sequence $3^{\prime}(1-1)$ that has less binding to TLS (Figure 4A), were tested to see binding profile for RBPs detected in these experiments with negative controls of $6 \mathrm{mer}$ and $9 \mathrm{mer}$ of the random RNA oligos. Binding experiments using sets of RNA oligos indicated that hnRNPUL2 bind all RNA oligos, suggesting its low specificity (Figure 4B). hnRNPU binds all RNA oligos except 6 mer of the randomized RNA oligos (Figure 4C). DDX21 has the similar profile to the hnRNPU, while hnRNPAB displays similar profile except less binding to 3'(1-1) (Figure 4C). hnRNPH1 and TLS has preferred bind to specific binding site, $5^{\prime}(1-1)$ (Figure 4D).

These data demonstrate that RNA binding is regulated with three factors, length, base compositions, and sequence of RNA (Figure 5AB). Furthermore, hnRNPU and hnRNPUL2 have low specificity binding to RNA. DDX21 and hnRNPAB have mild specificity, while TLS and hnRNPH1 exert high specificity binding. These different propensities of each RBP are thought to represent each specific biological role in living cells. 


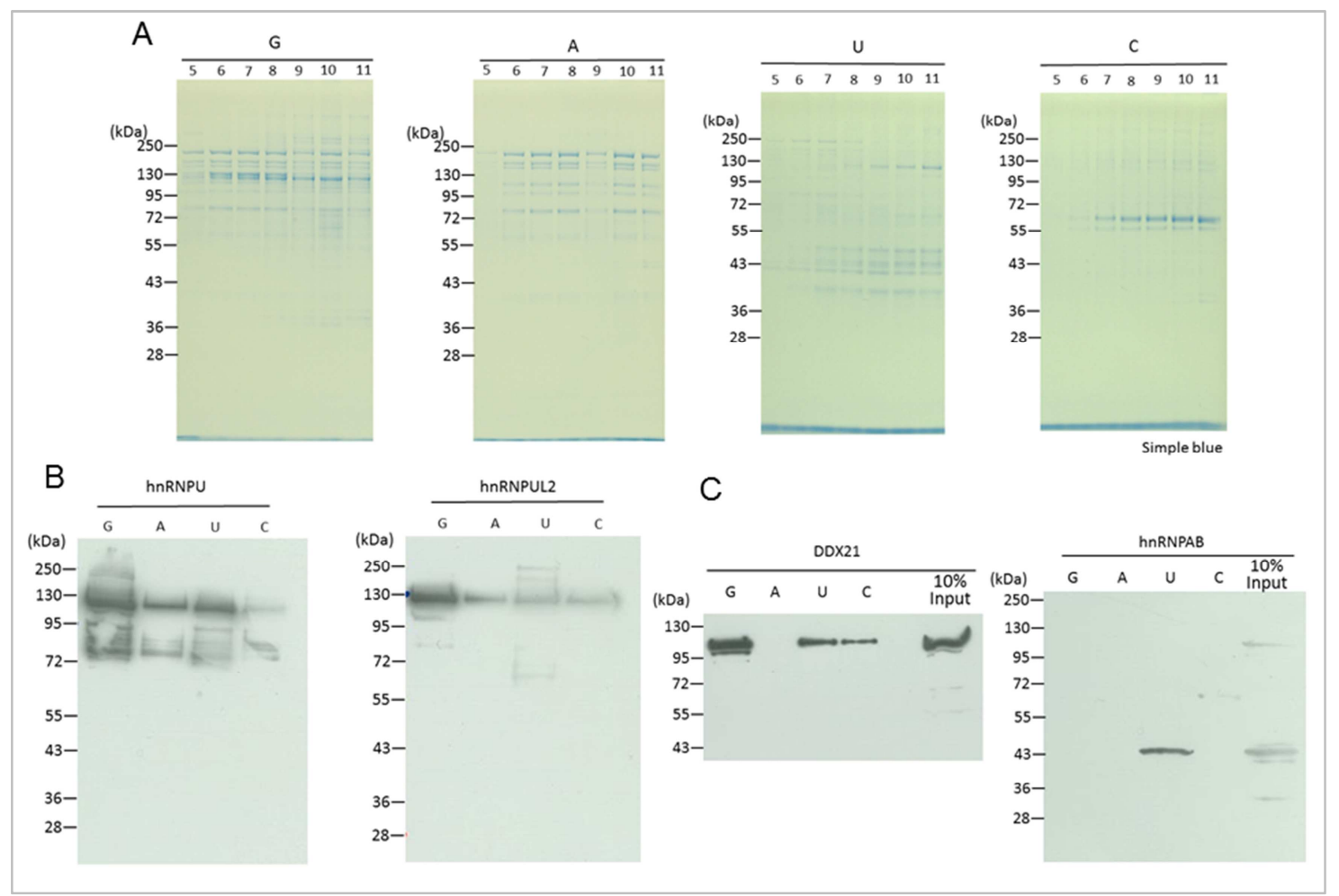

Figure 2. Binding profile of RNA oligos of GAUC from 5mers through 11mers.

(A) Distinct profiles are detected in each RNA oligo with each specific base composition (GAUC), and detected with CBB-staining. Fifty $\mu \mathrm{ls}$ of NE were used in Figure 2. "5" represents "5mer", and so on.

(B) hnRNPU and hnRNPUL2 bind RNA oligos with divergent base compositions. Length of oligos is fixed at 10mer.10\% inputs were similar intensity as DDX21 at Figure 2C (data not shown).

(C) DDX21 and hnRNPAB bind to RNA oligos with specific base compositions. Length of oligos is fixed at 10mer.

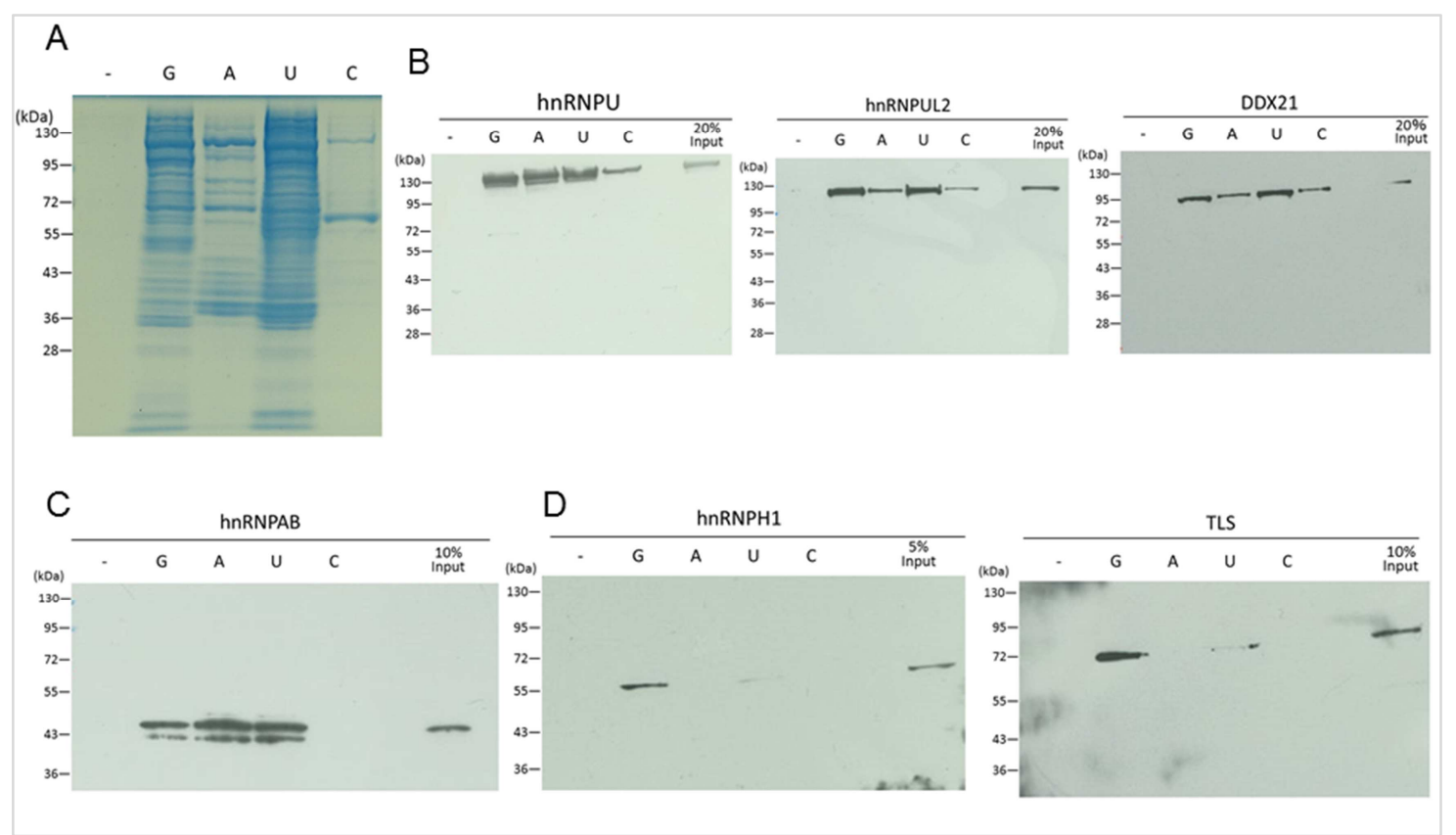

Figure 3. Bindings to Poly G, Poly A, Poly U, and Poly C RNA oligos (poly GAUC RNA oligos).

(A) Proteins are bound to poly GAUC RNA oligos, and detected with CBB-staining. Five $\mu$ ls of NE were used for experiments in Figure 3 .

(B) hnRNPU, hnRNPUL2, and DDX21 bind to all four Poly GAUC RNA oligos.

(C) hnRNPAB does not bind to poly C RNA oligos.

(D) hnRNPH1 and TLS have preferred binding to poly G RNA oligos. 


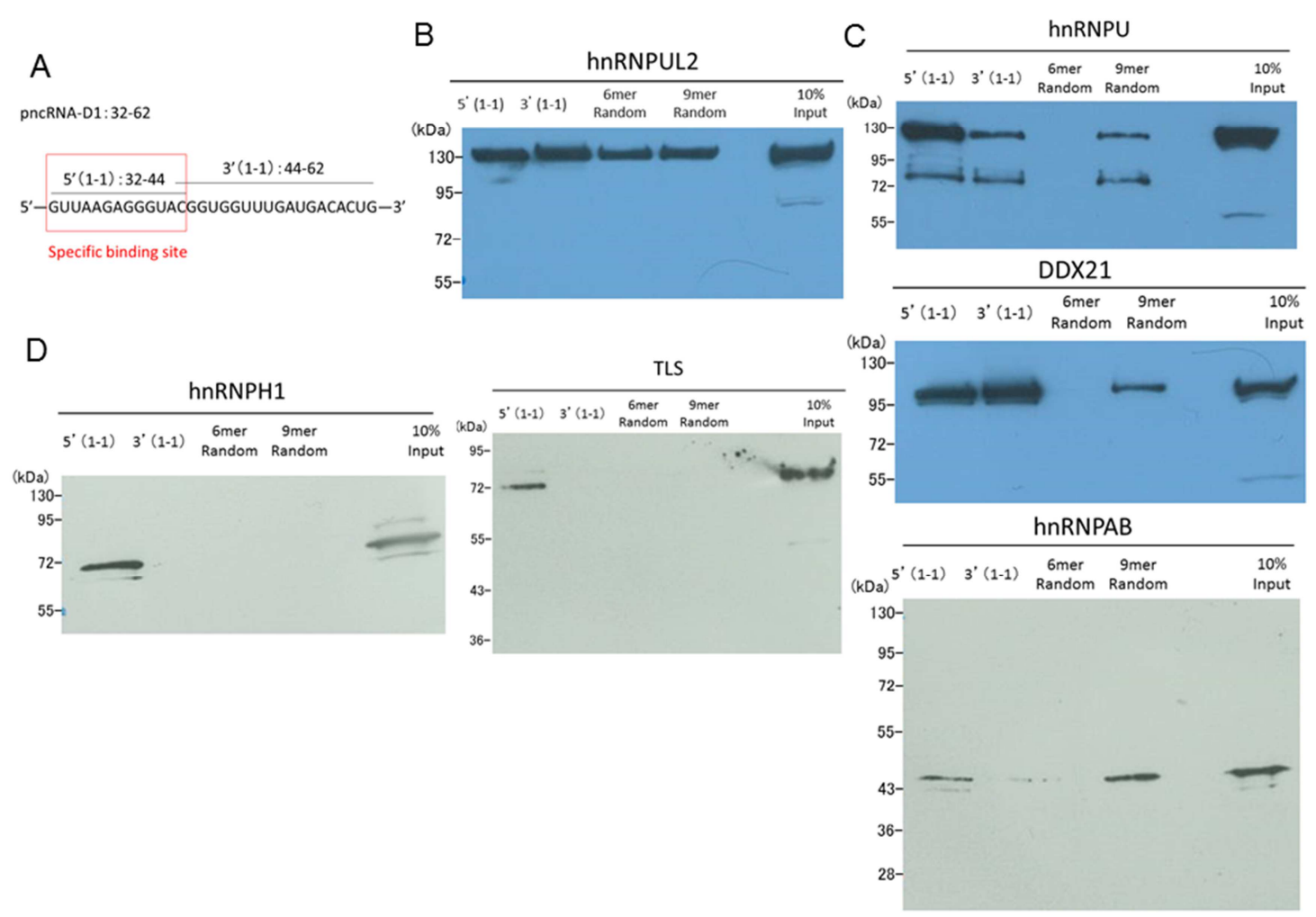

Figure 4. Specific and non-specific bindings of RBPS.

(A) pncRNA-D: 5'(1-1) and 3'(1-1) are depicted.

(B) hnRNPUL2 binds all four binding sites. Fifty $\mu \mathrm{ls}$ of NE were used in Figure 4.

(C) hnRNPU, DDX21, and hnRNPAB have similar binding profile.

(D) hnRNPH1 and TLS bind only high specific binding site, 5 '(1-1).

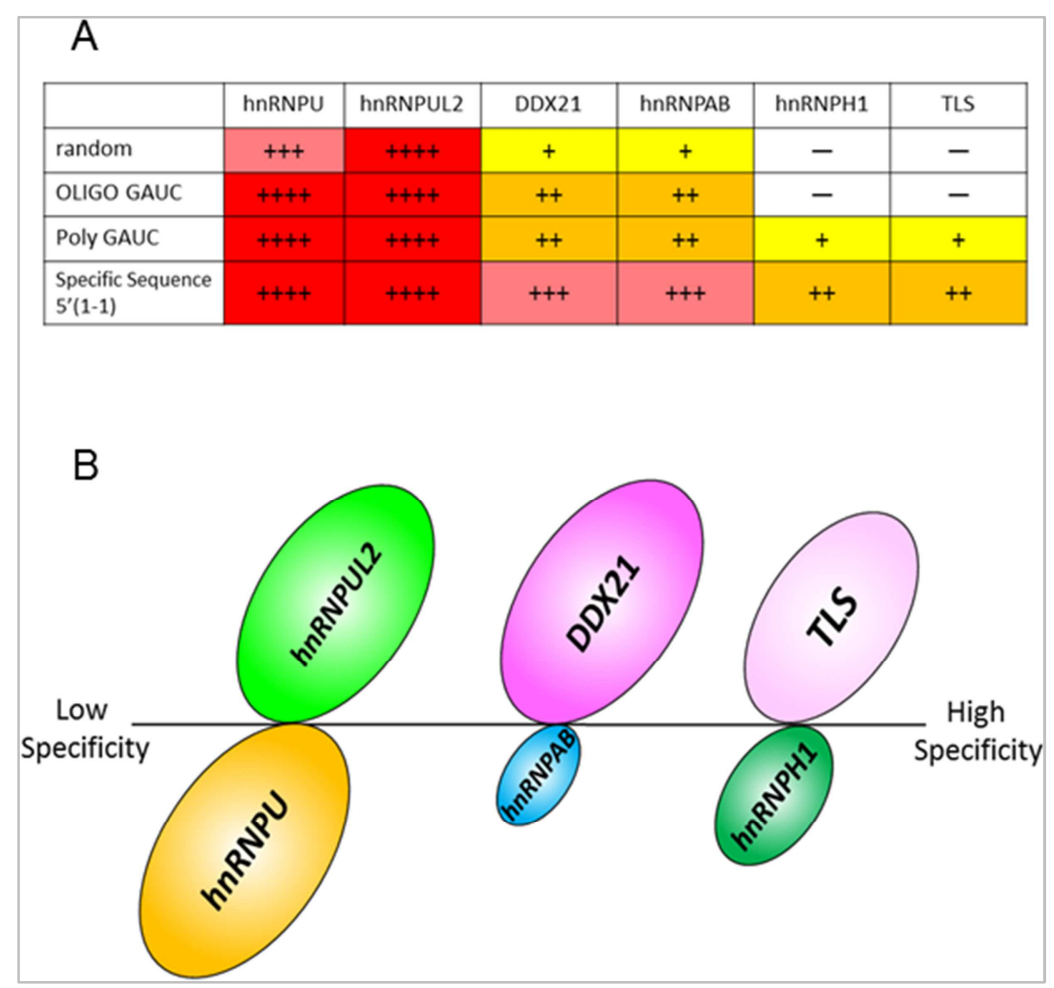

Figure 5. Length, base composition, and sequence of RNA determine affinity of RBPS.

(A) Summary of the binding assay.

(B) Visual summary of the propensity. 


\section{Discussion}

RNA binding is more complex task than DNA binding. DNA-binding protein is mostly transcription factor that recognizes specific responsive elements typically composed of consensus motifs with several DNA oligomers and regulates (in many cases, activates) gene expression [32-35]. It is crucial for DNA-binding transcription factor to precisely recognize specific binding sites. These molecules execute stringent regulations on transcription upon specific binding to response elements on the promoter or the enhancer region [36-38]. Therefore, high affinity of the DNA-binding proteins requires for their proper function.

Contrarily, RBPs are a versatile molecule and have more divergent functions like RNA splicing, modulating RNA metabolism, builder for cellular structures [39-42]. In some cases, RBPs just connect RNAs to proteins to form cellular structures [24, 42, 43]. Versatility of RBP functions endows wide ranges of affinity of their binding capacity [44-46]. RBPs are characterized by proficiency of RNA perception and possess divergent capability in living cells.

Our binding assays have effectively demonstrated that RBPs have distinctive subgroups classified with criteria of their binding affinity $[28,29]$. The experiments using random RNA oligos unpredictably indicated that hnRNPUL2 and hnRNPU bind to these RNA oligos which are typically used for negative control of RNA binding assays [28, 29, 47]. It has been reported that hnRNPUL2 is a part of RNA polymerase II general transcription factor complex [48] and also works on DNA repair processes [49, 50], indicating roles in forming nuclear structures. These processes just require grasping RNA as a chemical compound, but do not need to exert specific perception of RNA sequences. hnRNPU also works as backbone of various nuclear and cellular structures [43]. Secondly, hnRNPAB and DDX21 are found to have mild affinity to specific RNAs equipped with distinctive base compositions even they are relatively at short length. These two RBPs play each specific biological role in programs in live cells [51-53]. At the third category, we found hnRNPH1 and TLS which recognize a binding site, $5^{\prime}(1-1)$, located in pncRNA-D transcribed from the cyclin D1 gene promoter. Intriguingly, $5^{\prime}(1-1)$ has been identified as specific site for TLS [28-30]. Our experiments demonstrated that hnRNPH1 also binds the site specific to TLS. hnRNPH1 and TLS share the same tendency to binding RNA. These two RBPs only bind poly $\mathrm{G}$, but no binding to random RNA oligos and to GAUC RNA oligomers. So far these RNAs tested, poly $G$ alone is supposed to form a structure, $G$ quadruplex which might generate specific bindings of RBPs [54-56]. hnRNPH1 and TLS specifically recognize G quadruplex structures $[57,58]$.

These data demonstrate that RNA binding is regulated with three factors, length, base compositions and sequence of RNAs. Furthermore, hnRNPU and hnRNPUL2 have low specificity binding to RNAs. DDX21 and hnRNPAB have mild specificity to RNAs, while TLS and hnRNPH1 exert high specific binding. These different tendencies of each RBP are supposed to represent each specific biological role in living cells [51, 59, 60].

Molecular mechanisms exerting these distinct affinities of RBPs are next issues to be investigated for RNA biology. One of clues for breakthrough is LCDs of these RBPs. Function of LCDs just has been recognized for their importance in cellular and molecular contexts. LCDs might possess their specific properties in their distinct molecular environments in cells. Solution of the structures of RBPs could provide more profound understanding of their functions in biological programs.

\section{Conclusion}

In this manuscript, we have demonstrated that RNA binding is regulated with three factors, length, base composition, and sequence. Furthermore, hnRNPU and hnRNPUL2 have low specificity binding to RNAs. DDX21 and hnRNPAB has mild specificity to RNAs, while TLS and hnRNPH1 exert high specific binding. These different propensities in bindings of RBPs could represent specific biological roles in living cells.

\section{Acknowledgements}

The authors would thank for excellent technical supports by Mr. Sei-ichi Aikawa and Fumiko Matsuzawa (Altif Laboratories Inc.). This study was supported by Grant-in-Aid for Scientific Research (C: 18K06939). This work was also supported in part by a grant-in-aid for "Support Project of Strategic Research Center in Private Universities" from the Ministry of Education, Culture, Sports, Science and Technology (MEXT) to Saitama Medical University Research Center for Genomic Medicine.

\section{References}

[1] Kurokawa R (2015) Long Noncoding RNAs. In, pp. 257. Springer.

[2] Lipovich L, Tarca AL, Cai J, Jia H, Chugani HT, Sterner KN, Grossman LI, Uddin M, Hof PR, Sherwood CC, et al. (2014) Developmental changes in the transcriptome of human cerebral cortex tissue: long noncoding RNA transcripts. Cereb Cortex 24, 1451-1459, doi: 10.1093/cercor/bhs414.

[3] Derrien $\mathrm{T}$, Johnson R, Bussotti G, Tanzer A, Djebali S, Tilgner H, Guernec G, Martin D, Merkel A, Knowles DG, et al. (2012) The GENCODE v7 catalog of human long noncoding RNAs: analysis of their gene structure, evolution, and expression. Genome research 22, 1775-1789, doi: $10.1101 /$ gr.132159.111

[4] Carninci P \& Kasukawa T \& Katayama S \& Gough J \& Frith MC \& Maeda N \& Oyama R \& Ravasi T \& Lenhard B \& Wells C, et al. (2005) The transcriptional landscape of the mammalian genome. Science 309, 1559-1563, doi: 309/5740/1559 [pii] 10.1126/science. 1112014 . 
[5] Khalil AM, Guttman M, Huarte M, Garber M, Raj A, Rivea Morales D, Thomas K, Presser A, Bernstein BE, van Oudenaarden A, et al. (2009) Many human large intergenic noncoding RNAs associate with chromatin-modifying complexes and affect gene expression. Proc Natl Acad Sci U S A 106, 11667-11672, doi: 0904715106 [pii]10.1073/pnas.0904715106.

[6] Necsulea A, Soumillon M, Warnefors M, Liechti A, Daish T, Zeller U, Baker JC, Grutzner F \& Kaessmann H (2014) The evolution of IncRNA repertoires and expression patterns in tetrapods. Nature 505, 635-640, doi: 10.1038/nature12943.

[7] Chi KR (2016) Finding function in mystery transcripts. Nature 529, 423-425, doi: 10.1038/529423a.

[8] Kurokawa R (2012) Generation of Functional Long Noncoding RNA Through Transcription and Natural Selection. In Regulatory RNAs, pp. 151-174. Springer.

[9] Djebali S, Davis CA, Merkel A, Dobin A, Lassmann T, Mortazavi A, Tanzer A, Lagarde J, Lin W, Schlesinger F, et al. (2012) Landscape of transcription in human cells. Nature 489, 101-108, doi: 10.1038/nature11233.

[10] Hon C-C, Ramilowski JA, Harshbarger J, Bertin N, Rackham OJL, Gough J, Denisenko E, Schmeier S, Poulsen TM, Severin $\mathrm{J}$, et al. (2017) An atlas of human long non-coding RNAs with accurate 5' ends. Nature 543, 199-204, doi: 10.1038 /nature21374

http://www.nature.com/nature/journal/v543/n7644/abs/nature2 1374.html\#supplementary-information.

[11] Kurokawa R (2011) Long noncoding RNA as a regulator for transcription. Prog Mol Subcell Biol 51, 29-41, doi: 10.1007/978-3-642-16502-3 2.

[12] Kurokawa R (2011) Promoter-associated long noncoding RNAs repress transcription through a RNA binding protein TLS. Advances in experimental medicine and biology 722, 196-208, doi: 10.1007/978-1-4614-0332-6_12.

[13] Kurokawa R (2015) Initiation of Transcription Generates Divergence of Long Noncoding RNAs. In Long Noncoding RNAs, pp. 69-91. Springer.

[14] Kurokawa R, Rosenfeld MG \& Glass CK (2009) Transcriptional regulation through noncoding RNAs and epigenetic modifications. RNA Biol 6, 233-236, doi: 8329 [pii].

[15] Lunde BM, Moore C \& Varani G (2007) RNA-binding proteins: modular design for efficient function. Nat Rev Mol Cell Biol 8, 479-490, doi: 10.1038/nrm2178.

[16] Clery A, Blatter M \& Allain FH (2008) RNA recognition motifs: boring? Not quite. Current opinion in structural biology 18, 290-298, doi: 10.1016/j.sbi.2008.04.002.

[17] Linder P \& Jankowsky E (2011) From unwinding to clamping the DEAD box RNA helicase family. Nat Rev Mol Cell Biol 12, 505-516, doi: 10.1038/nrm3154.

[18] Ramakrishnan V (2014) The ribosome emerges from a black box. Cell 159, 979-984, doi: 10.1016/j.cell.2014.10.052.

[19] Steitz TA (2008) A structural understanding of the dynamic ribosome machine. Nat Rev Mol Cell Biol 9, 242-253, doi: $10.1038 / \mathrm{nrm} 2352$.

[20] Behrmann E, Loerke J, Budkevich TV, Yamamoto K, Schmidt
A, Penczek PA, Vos MR, Burger J, Mielke T, Scheerer P, et al. (2015) Structural snapshots of actively translating human ribosomes. Cell 161, 845-857, doi: 10.1016/j.cell.2015.03.052.

[21] Papasaikas P \& Valcarcel J (2016) The Spliceosome: The Ultimate RNA Chaperone and Sculptor. Trends in biochemical sciences 41, 33-45, doi: 10.1016/j.tibs.2015.11.003.

[22] Plaschka C, Lin PC \& Nagai K (2017) Structure of a pre-catalytic spliceosome. Nature 546, 617-621, doi: 10.1038 /nature22799.

[23] Wright PE \& Dyson HJ (2015) Intrinsically disordered proteins in cellular signalling and regulation. Nat Rev Mol Cell Biol 16, 18-29, doi: $10.1038 / \mathrm{nrm} 3920$.

[24] Castello A, Fischer B, Frese CK, Horos R, Alleaume AM, Foehr S, Curk T, Krijgsveld J \& Hentze MW (2016) Comprehensive Identification of RNA-Binding Domains in Human Cells. Molecular Cell 63, 696-710, doi: 10.1016/j.molcel.2016.06.029.

[25] Kwon SC, Yi H, Eichelbaum K, Fohr S, Fischer B, You KT, Castello A, Krijgsveld J, Hentze MW \& Kim VN (2013) The RNA-binding protein repertoire of embryonic stem cells. Nature structural \& molecular biology 20, 1122-1130, doi: 10.1038/nsmb.2638.

[26] Liepelt A, Naarmann-de Vries IS, Simons N, Eichelbaum K, Fohr S, Archer SK, Castello A, Usadel B, Krijgsveld J, Preiss T, et al. (2016) Identification of RNA-binding Proteins in Macrophages by Interactome Capture. Mol Cell Proteomics 15, 2699-2714, doi: 10.1074/mcp. M115.056564.

[27] Beckmann BM, Horos R, Fischer B, Castello A, Eichelbaum K, Alleaume AM, Schwarzl T, Curk T, Foehr S, Huber W, et al. (2015) The RNA-binding proteomes from yeast to man harbour conserved enigmRBPs. Nat Commun 6, 10127, doi: $10.1038 /$ ncomms 10127 .

[28] Cui W, Yoneda R, Ueda N \& Kurokawa R (2018) Arginine methylation of translocated in liposarcoma (TLS) inhibits its binding to long noncoding RNA, abrogating TLS-mediated repression of $\mathrm{CBP} / \mathrm{p} 300$ activity. The Journal of biological chemistry, doi: 10.1074/jbc. RA117.000598.

[29] Yoneda R, Suzuki S, Mashima T, Kondo K, Nagata T, Katahira M \& Kurokawa R (2016) The binding specificity of Translocated in LipoSarcoma/FUsed in Sarcoma with lncRNA transcribed from the promoter region of cyclin D1. Cell \& bioscience 6, 4, doi: 10.1186/s13578-016-0068-8.

[30] Wang X, Arai S, Song X, Reichart D, Du K, Pascual G, Tempst P, Rosenfeld MG, Glass CK \& Kurokawa R (2008) Induced ncRNAs allosterically modify RNA-binding proteins in cis to inhibit transcription. Nature 454, 126-130, doi: nature06992 [pii] 10.1038/nature06992.

[31] Song X, Wang X, Arai S \& Kurokawa R (2012) Promoter-associated noncoding RNA from the CCND1 promoter. Methods in molecular biology 809, 609-622, doi: 10.1007/978-1-61779-376-9_39.

[32] Roeder RG (1991) The complexities of eukaryotic transcription initiation: regulation of preinitiation complex assembly. Trends Biochem Sci 16, 402-408.

[33] Roeder RG (2003) Lasker Basic Medical Research Award. The eukaryotic transcriptional machinery: complexities and mechanisms unforeseen. Nature medicine 9, 1239-1244, doi: $10.1038 / \mathrm{nm} 938$. 
[34] Glass CK \& Rosenfeld MG (2000) The coregulator exchange in transcriptional functions of nuclear receptors. Genes Dev 14, 121-141.

[35] Rosenfeld MG, Lunyak VV \& Glass CK (2006) Sensors and signals: a coactivator/corepressor/epigenetic code for integrating signal-dependent programs of transcriptional response. Genes \& development 20, 1405-1428.

[36] Glass CK, Rosenfeld MG, Rose DW, Kurokawa R, Kamei Y, Xu L, Torchia J, Ogliastro MH \& Westin S (1997) Mechanisms of transcriptional activation by retinoic acid receptors. Biochem Soc Trans 25, 602-605.

[37] Kurokawa R, Soderstrom M, Horlein A, Halachmi S, Brown M, Rosenfeld MG \& Glass CK (1995) Polarity-specific activities of retinoic acid receptors determined by a co-repressor. Nature 377, 451-454, doi: 10.1038/377451a0.

[38] Kurokawa R, Yu VC, Naar A, Kyakumoto S, Han Z, Silverman S, Rosenfeld MG \& Glass CK (1993) Differential orientations of the DNA-binding domain and carboxy-terminal dimerization interface regulate binding site selection by nuclear receptor heterodimers. Genes Dev 7, 1423-1435.

[39] Dreyfuss G, Kim VN \& Kataoka N (2002) Messenger-RNA-binding proteins and the messages they carry. Nat Rev Mol Cell Biol 3, 195-205, doi: 10.1038/nrm760.

[40] Hudson WH \& Ortlund EA (2014) The structure, function and evolution of proteins that bind DNA and RNA. Nat Rev Mol Cell Biol 15, 749-760, doi: 10.1038/nrm3884.

[41] Cech TR \& Steitz JA (2014) The noncoding RNA revolution-trashing old rules to forge new ones. Cell 157, 77-94, doi: 10.1016/j.cell.2014.03.008.

[42] Hentze MW, Castello A, Schwarzl T \& Preiss T (2018) A brave new world of RNA-binding proteins. Nat Rev Mol Cell Biol 19, 327-341, doi: 10.1038/nrm.2017.130.

[43] Hasegawa Y, Brockdorff N, Kawano S, Tsutui K \& Nakagawa $\mathrm{S}$ (2010) The matrix protein hnRNPU is required for chromosomal localization of Xist RNA. Dev Cell 19, 469-476, doi: 10.1016/j.devcel.2010.08.006.

[44] Lambert N, Robertson A, Jangi M, McGeary S, Sharp PA \& Burge CB (2014) RNA Bind-n-Seq: quantitative assessment of the sequence and structural binding specificity of RNA binding proteins. Molecular Cell 54, 887-900, doi: 10.1016/j.molcel.2014.04.016.

[45] Zhou Q, Kunder N, De la Paz JA, Lasley AE, Bhat VD, Morcos F \& Campbell ZT (2018) Global pairwise RNA interaction landscapes reveal core features of protein recognition. Nat Commun 9, 2511, doi: 10.1038/s41467-018-04729-0.

[46] Jankowsky E \& Harris ME (2015) Specificity and nonspecificity in RNA-protein interactions. Nat Rev Mol Cell Biol 16, 533-544, doi: 10.1038/nrm4032.

[47] Khanam T, Muddashetty R, Kahvejian A, Sonenberg N \& Brosius J (2006) Poly(A)-Binding Protein Binds to A-Rich Sequences via RNA-Binding Domains $1+2$ and $3+4$. Vol. 3 .

[48] Kim MK \& Nikodem VM (1999) hnRNPU Inhibits Carboxy-Terminal Domain Phosphorylation by TFIIH and
Represses RNA Polymerase II Elongation. Molecular and Cellular Biology 19, 6833-6844.

[49] Berglund FM \& Clarke PR (2009) hnRNP-U is a specific DNA-dependent protein kinase substrate phosphorylated in response to DNA double-strand breaks. Biochemical and biophysical research communications 381, 59-64, doi: 10.1016/j.bbrc.2009.02.019.

[50] Polo SE, Blackford AN, Chapman JR, Baskcomb L, Gravel S, Rusch A, Thomas A, Blundred R, Smith P, Kzhyshkowska J, et al. (2012) Regulation of DNA-end resection by hnRNPU-like proteins promotes DNA double-strand break signaling and repair. Molecular Cell 45, 505-516, doi: 10.1016/j.molcel.2011.12.035.

[51] Song C, Hotz-Wagenblatt A, Voit R \& Grummt I (2017) SIRT7 and the DEAD-box helicase DDX21 cooperate to resolve genomic R loops and safeguard genome stability. Genes \& development 31, 1370-1381, doi: 10.1101/gad.300624.117.

[52] Calo E, Flynn RA, Martin L, Spitale RC, Chang HY \& Wysocka J (2015) RNA helicase DDX21 coordinates transcription and ribosomal RNA processing. Nature 518, 249-253, doi: 10.1038/nature13923.

[53] Khan FA, Jaiswal AK \& Szer W (1991) Cloning and sequence analysis of a human type A/B hnRNP protein. FEBS Letters 290, 159-161, doi: doi:10.1016/0014-5793(91)81249-8.

[54] Lane AN, Chaires JB, Gray RD \& Trent JO (2008) Stability and kinetics of G-quadruplex structures. Nucleic Acids Research 36, 5482-5515, doi: 10.1093/nar/gkn517.

[55] Booy EP, Meier M, Okun N, Novakowski SK, Xiong S, Stetefeld J \& McKenna SA (2012) The RNA helicase RHAU (DHX36) unwinds a G4-quadruplex in human telomerase RNA and promotes the formation of the $\mathrm{P} 1$ helix template boundary. Nucleic Acids Research 40, 4110-4124, doi: 10.1093/nar/gkr1306.

[56] Rhodes D \& Lipps HJ (2015) G-quadruplexes and their regulatory roles in biology. Nucleic Acids Research 43, 8627-8637, doi: 10.1093/nar/gkv862.

[57] Takahama K \& Oyoshi T (2013) Specific binding of modified RGG domain in TLS/FUS to G-quadruplex RNA: tyrosines in RGG domain recognize 2'-OH of the riboses of loops in G-quadruplex. J Am Chem Soc 135, 18016-18019, doi: $10.1021 / \mathrm{ja} 4086929$.

[58] Takahama K, Takada A, Tada S, Shimizu M, Sayama K, Kurokawa R \& Oyoshi T (2013) Regulation of telomere length by G-quadruplex telomere DNA- and TERRA-binding protein TLS/FUS. Chemistry \& biology 20, 341-350, doi: 10.1016/j.chembiol.2013.02.013.

[59] Li W, Notani D \& Rosenfeld MG (2016) Enhancers as non-coding RNA transcription units: recent insights and future perspectives. Nature reviews Genetics 17, 207-223, doi: 10.1038/nrg.2016.4.

[60] Hua JT, Ahmed M, Guo H, Zhang Y, Chen S, Soares F, Lu J, Zhou S, Wang M, Li H, et al. (2018) Risk SNP-Mediated Promoter-Enhancer Switching Drives Prostate Cancer through lncRNA PCAT19. Cell 174, 564-575 e518, doi: 10.1016/j.cell.2018.06.014. 